\title{
Cranial flap fixation in sheep using a resorbable bone adhesive
}

\author{
Kevin T. Foley, MD, ${ }^{1}$ Eric J. Woodard, MD, ${ }^{2}$ Jonathan R. Slotkin, MD, ${ }^{3}$ Cassandra K. Mayotte, MBA, ${ }^{4}$ \\ Abigail C. Baldwin, ${ }^{5}$ Michael C. Brown, ${ }^{5}$ and Brian J. Hess, MBA $^{5}$ \\ 1Department of Neurosurgery, University of Tennessee Health Science Center and Semmes Murphey Neurologic and Spine \\ Institute, Memphis, Tennessee; '2Department of Neurosurgery, New England Baptist Hospital, Boston, Massachusetts; \\ ${ }^{3}$ Department of Neurosurgery, Geisinger Health System, Danville, Pennsylvania; ${ }^{4}$ Stryker Corporation, Mahwah, New Jersey; \\ and ${ }^{5}$ LaunchPad Medical, Lowell, Massachusetts
}

OBJECTIVE The authors' goal in this study was to investigate the use of a novel, bioresorbable, osteoconductive, wetfield mineral-organic bone adhesive composed of tetracalcium phosphate and phosphoserine (TTCP-PS) for cranial bone flap fixation and compare it with conventional low-profile titanium plates and self-drilling screws.

METHODS An ovine craniotomy surgical model was used to evaluate the safety and efficacy of TTCP-PS over 2 years. Bilateral cranial defects were created in 41 sheep and were replaced in their original position. The gaps (kerfs) were completely filled with TTCP-PS (T1 group), half-filled with TTCP-PS (T2 group), or left empty and the flaps fixated by plates and screws as a control (C group). At 12 weeks, 1 year, and 2 years following surgery, the extent of bone healing, local tissue effects, and remodeling of the TTCP-PS were analyzed using macroscopic observations and histopathological and histomorphometric analyses. Flap fixation strength was evaluated by biomechanical testing at 12 weeks and 1 year postoperatively.

RESULTS No adverse local tissue effects were observed in any group. At 12 weeks, the bone flap fixation strengths in test group $1(1689 \pm 574 \mathrm{~N})$ and test group $2(1611 \pm 501 \mathrm{~N})$ were both statistically greater $(p=0.01)$ than that in the control group $(663 \pm 385 \mathrm{~N})$. From 12 weeks to 1 year, the bone flap fixation strengths increased significantly $(p<0.05)$ for all groups. At 1 year, the flap fixation strength in test group $1(3240 \pm 423 \mathrm{~N})$ and test group $2(3212 \pm 662 \mathrm{~N})$ were both statistically greater $(p=0.04$ and $p=0.02$, respectively) than that in the control group $(2418 \pm 1463 \mathrm{~N})$; however, there was no statistically significant difference in the strengths when comparing the test groups at both timepoints. Test group 1 had the best overall performance based on histomorphometric evaluation and biomechanical testing. At 2 years postoperatively, the kerfs filled with TTCP-PS had histological evidence of osteoconduction and replacement of TTCP-PS by bone with nearly complete osteointegration.

CONCLUSIONS TTCP-PS was demonstrated to be safe and effective for cranial flap fixation in an ovine model. In this study, the bioresorbable, osteoconductive bone adhesive appeared to have multiple advantages over standard plateand-screw bone flap fixation, including biomechanical superiority, more complete and faster bony healing across the flap kerfs without fibrosis, and the minimization of bone flap and/or hardware migration and loosening. These properties of TTCP-PS may improve human cranial bone flap fixation and cranioplasty.

https://thejns.org/doi/abs/10.3171/2019.11.JNS192806

KEYWORDS bone adhesive; flap fixation; bioresorbable; osteoconduction; osteointegration; fibrosis; surgical technique

$\mathrm{T}$ ITANIUM plates and screws are the current state-ofthe-art devices for cranial bone flap fixation. They occasionally loosen, resulting in hardware protrusion, cosmetic disfiguration, or pain, and can require reoperation..$^{5,9}$

Bone cements have been suggested as an alternative to metal for cranial fixation, but existing cements have not replaced hardware, primarily because they lack sufficient strength for this application. ${ }^{4}$ Currently available cements are also not chemically adhesive, do not provide a watertight seal, and add significant cost to the procedure.

During the initial healing process following cranial

ABBREVIATIONS TTCP-PS = tetracalcium phosphate and phosphoserine.

SUBMITTED October 14, 2019. ACCEPTED November 25, 2019.

INCLUDE WHEN CITING Published online February 7, 2020; DOI: 10.3171/2019.11.JNS192806. 


\begin{tabular}{|c|c|c|c|c|c|c|}
\hline \multirow[b]{3}{*}{ Group } & \multirow[b]{3}{*}{ Fixation Technique } & \multicolumn{5}{|c|}{ Timepoint } \\
\hline & & \multicolumn{2}{|r|}{$12 \mathrm{Wks}$} & \multicolumn{2}{|r|}{$1 \mathrm{Yr}$} & \multirow{2}{*}{$\begin{array}{c}2 \text { Yrs } \\
\text { Histopath \& Histomorph } \\
\text { Analyses }\end{array}$} \\
\hline & & $\begin{array}{l}\text { Biomechanical } \\
\text { Testing }\end{array}$ & $\begin{array}{c}\text { Histopath \& Histomorph } \\
\text { Analyses }\end{array}$ & $\begin{array}{l}\text { Biomechanical } \\
\text { Testing }\end{array}$ & $\begin{array}{c}\text { Histopath \& Histomorph } \\
\text { Analyses }\end{array}$ & \\
\hline $\mathrm{T} 1$ & TTCP-PS, full application & 6 sites & 6 sites & 7 sites & 4 sites & 4 sites \\
\hline $\mathrm{T} 2$ & TTCP-PS, half application & 6 sites & 7 sites & 5 sites & 4 sites & 4 sites \\
\hline Control & Plates \& screws & 6 sites & 7 sites & 4 sites & 2 sites & 4 sites \\
\hline
\end{tabular}

Histomorph = histomorphological; histopath = histopathological; T1 = test group 1; T2 = test group 2.

surgery, CSF can leak through the dural closure and escape beneath the scalp flap through the craniotomy margin, establishing a route for bacterial infection. ${ }^{2} \mathrm{CSF}$ leaks and infections are potentially devastating complications that often result in hospitalization and can even lead to death. ${ }^{2,6-8,10,13}$ While infection and CSF leaks are responsible for the majority of reoperations following craniotomy, pain and protrusion related to cranial plates and screws have been reported to prompt reoperation in $1.6 \%$ of cases. ${ }^{5}$

Composed of a wet-field self-setting aqueous-based reaction comprising tetracalcium phosphate and phosphoserine, TTCP-PS is a novel, bioresorbable bone adhesive. When initially mixed, TTCP-PS maintains a tackyadhesive character through its initial setting process. It cures within minutes after application and produces a durable adhesive with load-bearing bond strength to wet bone tissue and metals. TTCP-PS has been studied in a canine model for dental implant stabilization ${ }^{1}$ and was recently approved by the Food and Drug Administration for first-in-human testing for this application. The objective of this study was to investigate the safety and efficacy of TTCP-PS for cranial bone flap fixation in comparison with titanium plates and screws in an ovine surgical model.

\section{Methods}

\section{Mineral-Organic Bone Adhesive}

TTCP-PS (Tetranite Bone Adhesive; LaunchPad Medical) was provided by the manufacturer (Stryker) packaged and sealed in powdered form in bowls and sterilized by gamma irradiation (20-30 kGy). The powdered material is composed of calcium phosphate, primarily tetracalcium phosphate (TTCP) phase, and phosphoserine (PS). It is mixed with sterile water for injection at a powder-to-liquid ratio of $3.9 \mathrm{wt} / \mathrm{vol}$.

\section{Fixation Hardware}

The commercially available bone flap fixation system comprised 1) titanium low-profile 2-hole, 12-mm-long plates ( $\mathrm{n}=3$, Stryker) and 2) $1.5 \times 4-\mathrm{mm}$ self-drilling titanium screws $(\mathrm{n}=6$, Stryker).

\section{Animals}

Forty-one female sheep (50 to $75 \mathrm{~kg}$, age $>2$ years) were used in this study. The animals were kept in a purpose-designed room and fed a standard laboratory diet.
Before the experiment, the animals underwent a quarantine period to help ensure health and acclimation. Animal management conditions complied with the European requirements (EEC Directive 76/609), and the Biomatech ethics committee approved the protocol. Biomatech is an accredited facility and is registered with the French Department of Agriculture for animal housing, care, and investigations.

\section{Study Design Summary}

This study followed a 3-arm, controlled, randomized, prospective design. Two bilateral flaps were surgically created in the parietal region of the skull in each sheep (2 test sites per sheep). Each flap was then removed, replaced in its original position, and fixed in one of 3 ways. In test group 1, the full circumference of the kerf was completely filled with TTCP-PS. In test group 2, only half of the kerf was filled with TTCP-PS (one-quarter circumference on one side, a second quarter opposite the first). In the control group, the flap was fixed using three 2-hole titanium plates and 6 screws, and the kerf was left empty. At specific timepoints, tissues were harvested, and all sites were used either for mechanical testing or histological examination. The 3 groups were randomly assigned and allocated by permutation (Table 1).

\section{Craniotomy Surgical Procedure}

Premedication and anesthesia were administered by intravenous injection of a thiopental-pentobarbital mixture and atropine followed by inhalation of an $\mathrm{O}_{2}$ isoflurane mixture. Each sheep received preoperative analgesic treatment as well as prophylactic antibiotics. A midline incision was made through the skin from the orbits to the external occipital protuberance. The temporalis muscles were elevated from the frontal and parietal bones and retracted bilaterally. All remaining soft tissue was removed from the exposed site. Bilateral craniotomies were located at a distance of approximately $8 \mathrm{~mm}$ from the midline and $6 \mathrm{~mm}$ behind the frontal suture. Trephine drills were used to create circular cuts with a $15-\mathrm{mm}$ inner diameter, $19-\mathrm{mm}$ outer diameter, and an approximately 4-mm depth through the cranium, depending on skull thickness. Bone flaps were marked with crosshairs, and quadrants were labeled to provide correct flap orientation when the flaps were repositioned and fixed (Fig. 1). Sharp bony edges were smoothed with burrs. Flap thickness, position, diameter, and kerf length 


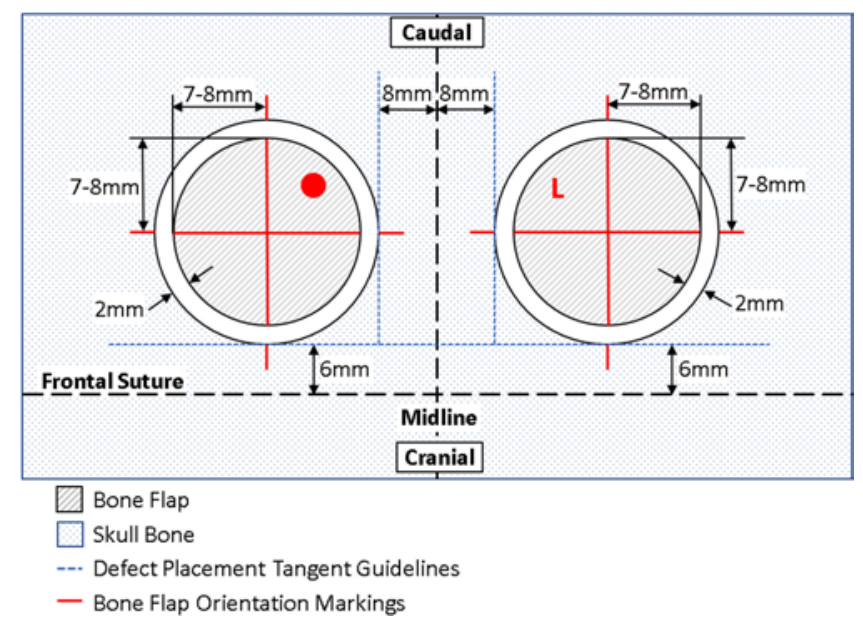

FIG. 1. Defect placement guidelines and bone flap orientation markings.

were measured and recorded. Bone flaps were immersed in sterile saline during the procedure before replacement. Craniotomy was repeated for the contralateral defect.

\section{Hardware Control Procedure}

After the bone flaps were dried, three 2-hole plates were fixed to the flaps with self-drilling screws. Each flap was replaced in its original position, and the remaining screws were used to secure the plates to the surrounding skull bone. Each plate was positioned with approximately $120^{\circ}$ spacing from the other (Fig. 2A).

\section{Test Group 1 Procedure (full application of TTCP-PS)}

The bone flap was dried and replaced in its original position. TTCP-PS was mixed with $2 \mathrm{~mL}$ of sterile water for injection using a spatula. After 30 seconds of mixing, the material became a paste and was transferred into a 3 -mL syringe; injection was performed within 2 minutes, 30 seconds from the beginning of mixing. TTCP-PS was injected starting at the bottom of the defect, ensuring full coverage through the entire thickness and all edges of the kerf. If necessary, additional TTCP-PS was applied to the kerf to fill any remaining gaps and to ensure that the TTCP-PS was flush with the surrounding bone. A gloved finger, moistened with sterile saline, was used to smooth the surface of the TTCP-PS to the surrounding bone. Excess TTCP-PS was removed with a plastic spatula. Sterile $0.9 \% \mathrm{NaCl}$ solution was applied at 5 minutes to evenly moisten the defect to prevent it from drying out. After 10 minutes, TTCP-PS had sufficiently set for soft-tissue closure (Fig. 2B).

\section{Test Group 2 Procedure (half application of TTCP-PS)}

Test group 2 was prepared in an identical manner as test group 1. However, instead of filling the entire kerf with TTCP-PS, the material was only applied to 2 opposite quarters of the kerf space. During material delivery, TTCP-PS was blocked from flowing into the 2 empty, opposing quarters by polyurethane stoppers that were placed into the kerf. The stoppers were removed within 2 minutes, 30 seconds. Sterile $0.9 \% \mathrm{NaCl}$ solution was applied at 5 minutes to evenly moisten the defect to prevent it from drying out. After 10 minutes, TTCP-PS had sufficiently set for soft-tissue closure (Fig. 2C).

For all treatment groups, the soft tissues were reapproximated over the defect sites. Povidone iodine solution was applied, and subcutaneous tissues were closed using a continuous suture with absorbable thread; the skin was closed using a continuous suture with a nonabsorbable thread. The skin was disinfected with oxytetracycline.

\section{Clinical Observations}

The health of all animals was monitored daily for any adverse reactions. When any clinical abnormality was detected, a complete veterinary clinical examination was conducted, and the observations were recorded. A veterinarian examined sheep showing severe signs of morbidity, and those animals were euthanized if needed. When premature euthanasia was necessary before termination of the study, a necropsy was performed. Sheep were also observed for signs of neurological effects before treatment, on postoperative days $1,2,3$, and 7; week 6 ; and at termination. Attention was given, but not limited, to mental state, weakness, locomotion/mobility, tremors/ seizures, circling, posture, head tilt, and ocular movements.

\section{Euthanasia}

At the end of the specified in vivo period, the animals were weighed and euthanized by lethal intravenous injection of pentobarbital solution (Dolethal, Vetoquinol).
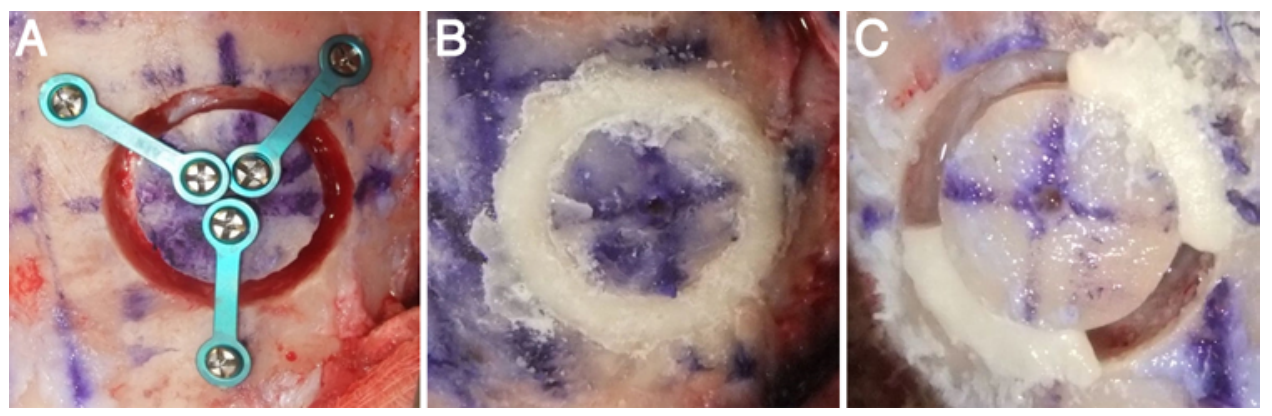

FIG. 2. Representative macroscopic images of each test group condition. A: Control group. B: Test group 1. C: Test group 2. 


\section{Necropsy}

The skin and underlying fascia were cut to visualize the surgical sites. Craniotomy sites were evaluated for evidence of flap movement from original positions, for flap fixation, for TTCP-PS presence in areas other than within the kerf, and disintegration or breakdown of the TTCP-PS. For sheep designated for histology and histomorphometry, the craniotomy sites with the surrounding bone were carefully removed while avoiding damage to the underlying brain tissue. The dura and surface of the brain in the region of the craniotomies were macroscopically observed, and any abnormality was recorded. The sites designated for histopathology were cut with approximately $1 \mathrm{~cm}$ of skull bone tissue surrounding the site and fixed in $10 \%$ neutral-buffered formalin. The sites designated for biomechanical testing were not separated but sampled together with the entire skinned skull intact. The explanted skulls were wrapped in saline-soaked gauze, sealed in plastic bags, and refrigerated between $4^{\circ} \mathrm{C}$ and $8^{\circ} \mathrm{C}$ until biomechanical testing.

\section{Cranial Flap Fixation Evaluation and Biomechanical Testing}

At necropsy, bone flap fixation quality was visually evaluated in a semiquantitative fashion using a bone flap fixation score. Observations of obvious screw loosening and dislodgment were also recorded. The fixation score was recorded as follows: No flap fixation, 0 ; severe flap movements, 1; moderate flap movements, 2; slight flap movements, 3; and no flap movements, 4 .

Biomechanical evaluation of cranial flap fixation was assessed through pushout testing completed by Stryker personnel at the Biomatech facility within 24 hours of skull explantation. Each skull was secured in a custom fixture to ensure that each bone flap site was oriented such that it was orthogonal in alignment with the application of force upon testing. Pushout testing was completed with a Tinius Olsen machine and a 5-kN load cell at a crosshead speed of $2 \mathrm{~mm} / \mathrm{min}$. The load cell was connected to a mechanical test adaptor, which made direct contact with the bone flap to execute the pushout test; this adaptor consisted of a metal cylinder with 3 protruding screws such that loads were applied only to bone flaps rather than to titanium hardware or TTCP-PS. Flap fixation strength was tested to failure. The maximum force reached for each bone flap was recorded as the fixation strength in Newtons (N).

\section{Histological Preparation}

Samples were prepared and labeled so that the histopathologist was blinded to treatment group. After complete fixation, implanted sites were dehydrated in alcohol solutions of increasing concentrations, cleared in xylene, and embedded in polymethylmethacrylate resin. Four sections per site were prepared. First, 2 paracentral sections of 20to $30-\mu \mathrm{m}$ thickness were obtained by a microcutting and grinding technique adapted from Donath et al. ${ }^{3}$ The 2 remaining blocks were glued together and cut, and 2 paracentral perpendicular sections were then prepared. The ground-section slides were stained with modified Paragon for histological analysis by means of bright-field microscopic evaluation. The bone tissues and surrounding soft tissues were evaluated using an analysis adapted from the ISO 10993-6 histopathology standard. Histomorphometric quantitative evaluation of the bone sites was conducted by scanning and examining the sites using a motorized microscope equipped with a color-images analyzing system (Samba, version 4.27; Samba Technologies).

\section{Statistical Analysis}

The mean and SD for the pushout strength and bone flap fixation score are reported by timepoint and test group. The mean is reported for histomorphometry by timepoint and test group. For testing the biomechanical significance, the mean pushout strength differences between timepoints and test groups were evaluated with a linear mixed-model analysis. Specifically, a model was used with both a subject-specific intercept and variances, which were allowed to differ by timepoint and test group. Estimated differences and $p$ values are reported. All analyses were conducted with NCSS version 11.0.2 software.

\section{Results \\ Clinical Observations}

The first 8 sheep operated on displayed motion difficulties after surgery due to surgical positioning, which was corrected in all remaining test animals. One sheep displayed transient abnormal neurological signs (tremors, circling, head tilt, and eye and lid impaired movements) that resolved in 24 hours. This animal sustained a dural laceration at surgery with intraoperative CSF leak. Another sheep was found dead 50 weeks after surgery. The cause of death was a large abscess surrounding the pharynx. No other abnormal clinical abnormalities were observed.

\section{Macroscopic Observations}

The only sites that demonstrated bone flap movement were sites in the control group at both 1 and 2 years. Screw loosening appeared to contribute to this, as loose screws were observed in 4 of 6 sites at 1 year and 6 of 7 sites at 2 years. In 3 of 7 sites in the control group, screws had completely backed out and were found in the surrounding soft tissues at 2 years. Test groups 1 and 2 showed no diffusion, disintegration, or breakage of the material at any point during the study. No bone flaps fixated with TTCPPS became mobile over the 2-year study period (Table 2).

\section{Mechanical Testing}

At each timepoint tested for biomechanical strength, the mean pushout strength of the flaps fixated with TTCPPS exceeded that of the flaps fixated with metal plates and screws (Table 3 and Fig. 3). At 12 weeks, the bone flap fixation strengths in test group $1(1689 \pm 574 \mathrm{~N})$ or test group $2(1611 \pm 501 \mathrm{~N})$ were both statistically greater $(\mathrm{p}=$ $0.01)$ than that in the control group $(663 \pm 385 \mathrm{~N})$. From 12 weeks to 1 year, the bone flap fixation strengths increased significantly $(\mathrm{p}<0.05)$ for all groups. At 1 year, the flap fixation strengths for test group $1(3240 \pm 423 \mathrm{~N})$ and test group $2(3212 \pm 662 \mathrm{~N})$ were both statistically greater $(\mathrm{p}$ 
TABLE 2. Summary of flap fixation score and behavior of sites

\begin{tabular}{|c|c|c|c|c|c|}
\hline Group & Timepoint & Mean \pm SD Score for Bone Flap Fixation* & Sites w/ Screw Loosening & Sites w/ Screw Migration & Sites w/ Mobile Plates \\
\hline \multirow{3}{*}{ Control } & 12 wks $(n=12)$ & \multirow{3}{*}{$2.7 \pm 0.4$} & 0 & 0 & 0 \\
\hline & $1 \mathrm{yr}(\mathrm{n}=6)$ & & 4 & 0 & 1 \\
\hline & 2 yrs $(n=7)$ & & 6 & 3 & 5 \\
\hline \multirow{3}{*}{$\mathrm{T} 1$} & 12 wks $(n=12)$ & & \multirow{3}{*}{\multicolumn{3}{|c|}{ Not applicable }} \\
\hline & $1 \mathrm{yr}(\mathrm{n}=7)$ & $3.9 \pm 0.4$ & & & \\
\hline & 2 yrs $(n=8)$ & & & & \\
\hline \multirow{3}{*}{ T2 } & 12 wks $(n=7)$ & & \multirow{3}{*}{\multicolumn{3}{|c|}{ Sites fixated w/ TTCP-PS w/o hardware }} \\
\hline & $1 \mathrm{yr}(\mathrm{n}=7)$ & $3.7 \pm 0.8$ & & & \\
\hline & 2 yrs $(n=5)$ & & & & \\
\hline
\end{tabular}

* 0 , no flap fixation; 1 , severe flap movements; 2 , moderate flap movements; 3 , slight flap movements; 4 , no flap movements.

$=0.04$ and $\mathrm{p}=0.02$, respectively) than that in the control group $(2418 \pm 1463 \mathrm{~N})$; however, there was no statistically significant difference in the strengths when comparing test groups 1 and 2 at both timepoints.

\section{Histological Examination}

In the histology images (Fig. 4), the bone is pink, the TTCP-PS implant is black, and the soft tissue is blue. The blue soft tissue can be seen in the kerf of the hardware control through 2 years; the degree of fibrosis in this group was consistently high. In contrast, the TTCP-PS groups had minimal fibrosis. Test group 1 exhibited no soft tissue in the kerf at any point, and new pink bone can be seen propagating across the kerf and through the implant material. This histology also shows the black implant material contacting nearly all of the surrounding bone, indicating a high level of osteointegration. The TTCP-PS also demonstrates resorption over time as the black becomes replaced with new, pink bone.

\section{Microscopic Observations at 12 Weeks Control Group}

Kerfs were mostly filled with a dense fibroconnective tissue, which was slightly infiltrated with macrophages. A variable amount of newly formed bone emerged from the kerf margins. Complete bone healing was never observed within the entire circular kerf of the sites.

\section{Test Group 1}

TTCP-PS appeared as a homogeneous material that had a moderate level of osteointegration. Some newly formed bone and signs of osteoconduction were observed within

TABLE 3. Cranial flap pushout strength

\begin{tabular}{ccc}
\hline & \multicolumn{2}{c}{ Strength $(\mathrm{N})$} \\
\cline { 2 - 3 } Group & 12 Wks $(n=6)$ & $1 \mathrm{Yr}(\mathrm{n}=4)$ \\
\hline T1 & $1689 \pm 574$ & $3240 \pm 423$ \\
\hline T2 & $1611 \pm 501$ & $3212 \pm 662$ \\
\hline Control & $663 \pm 385$ & $2418 \pm 1463$ \\
\hline
\end{tabular}

Values are presented as the mean \pm SD. the TTCP-PS. There were multinucleated giant cells and activated macrophages in the vicinity of the TTCP-PS, signifying signs of TTCP-PS resorption in conjunction with bone ingrowth. No evidence of cytotoxicity or osteolysis was detected.

\section{Test Group 2}

In areas in which TTCP-PS was present, observations were similar to those of test group 1. In areas in which the kerfs were empty, the tissue region was fibrotic and similar to that in the control group.

\section{Microscopic Observations at 1 Year \\ Control Group}

Moderate bone formation was noticed in the kerfs with slight osteoblast activity. Fibroconnective tissue filled the remaining space of the kerfs. Some macrophages were observed within this fibrous tissue.

\section{Test Group 1}

TTCP-PS was markedly osteointegrated with signs of TTCP-PS replacement by bone and osteoconduction. Some inflammatory infiltrates, including macrophages and multinucleated giant cells, were found in the lacunae of the new bone forming in the kerf. No evidence of cytotoxicity or osteolysis was detected.

\section{Test Group 2}

In the areas in which TTCP-PS was present, observations were similar to those of test group 1. In areas where the kerfs were empty, a higher level of bone healing was noted when compared with the control group (8 of 16 kerfs were healed in test group 1 in comparison with 0 of 16 in the control group). The tissue reaction in the nonhealed kerfs was fibrotic and similar to that observed in the control group.

\section{Microscopic Observations at 2 Years Control Group}

Kerfs were completely healed in 13 of 32 sections. Some osteoblast activity was noted. Fibrous tissue filled the remaining space of the non-fully healed kerfs. Some macrophages were observed within the fibroconnec- 
Foley et al.
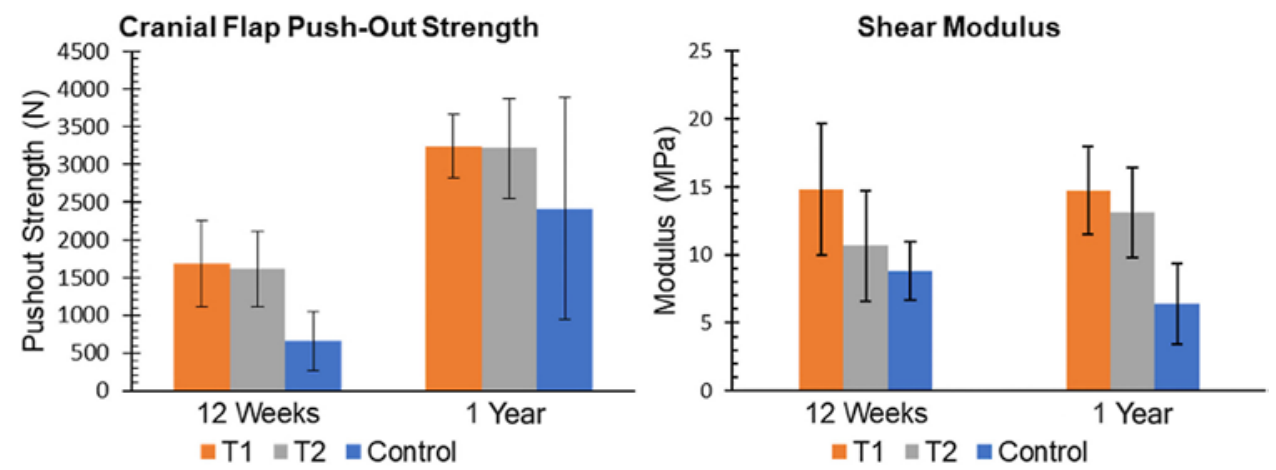

FIG. 3. Pushout strength and shear moduli of flaps fixated with TTCP-PS and hardware. $T 1=$ test group 1; T2 = test group 2 .

tive tissue, and signs of osteolysis were noted around the screws.

\section{Test Group 1}

TTCP-PS was nearly completely osteointegrated with signs of TTCP-PS replacement by bone and osteoconduction. No inflammatory infiltrates were found, and there was no evidence of cytotoxicity or osteolysis.

\section{Test Group 2}

In the areas in which TTCP-PS was present, observations were similar to those in the $\mathrm{T} 1$ group. In the areas where the kerf was empty, the tissue reaction was similar to that observed with the control group. There were moderate signs of material degradation.

\section{Histomorphometric Analysis}

Histomorphometric analysis was done to measure the total area of implant (TTCP-PS), bone, and soft tissue in a representative cross-section of each kerf (Fig. 5). The first 3 bars track test group 1, showing the resorption of TTCP-PS as it decreased at each timepoint and was replaced with new bone. The amount of soft tissue in the TTCP-PS groups was minimal, almost always below 5\%

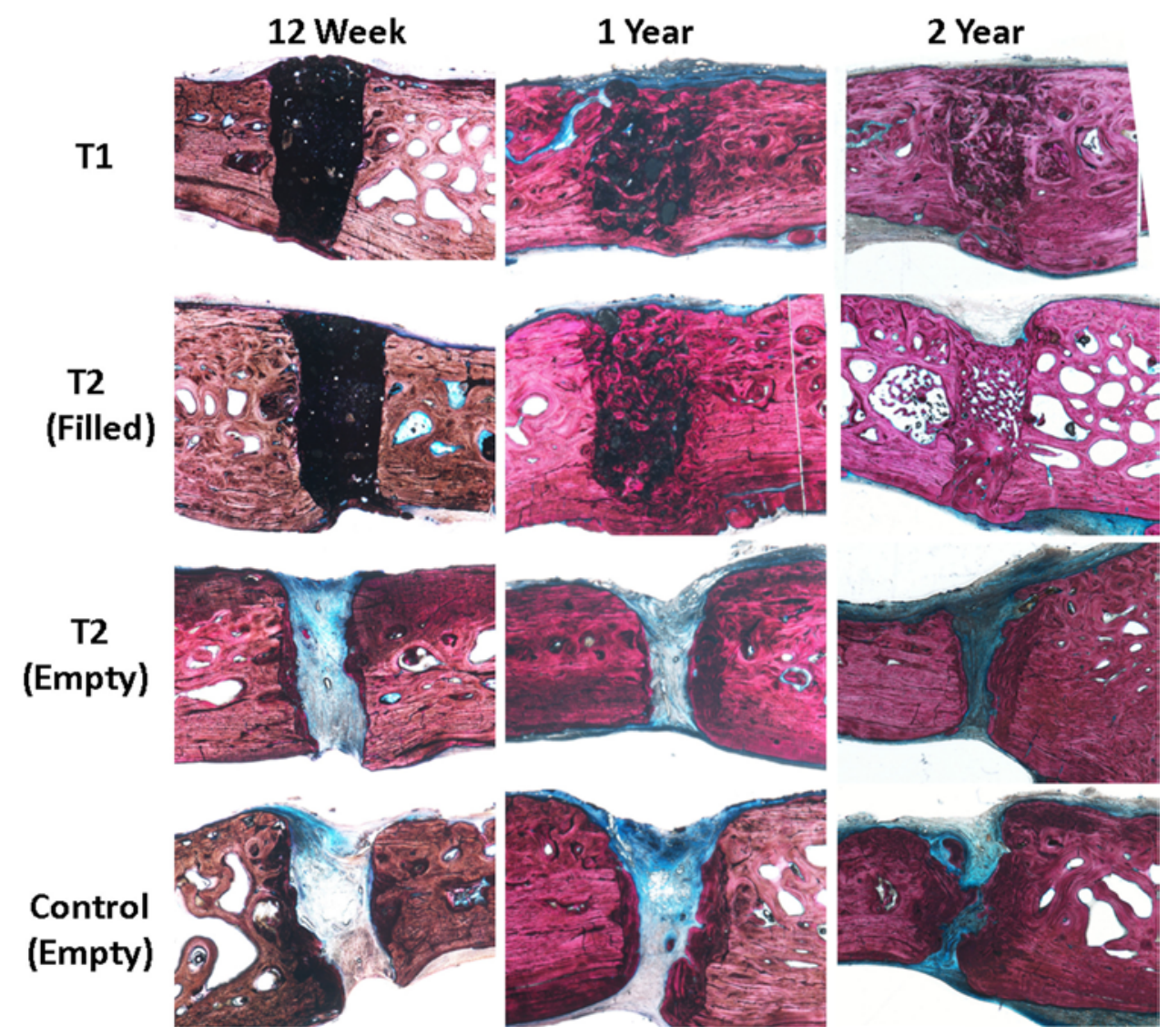

FIG. 4. Representative histological images obtained at all timepoints. Modified Paragon stain, original magnification $\times 20$. 


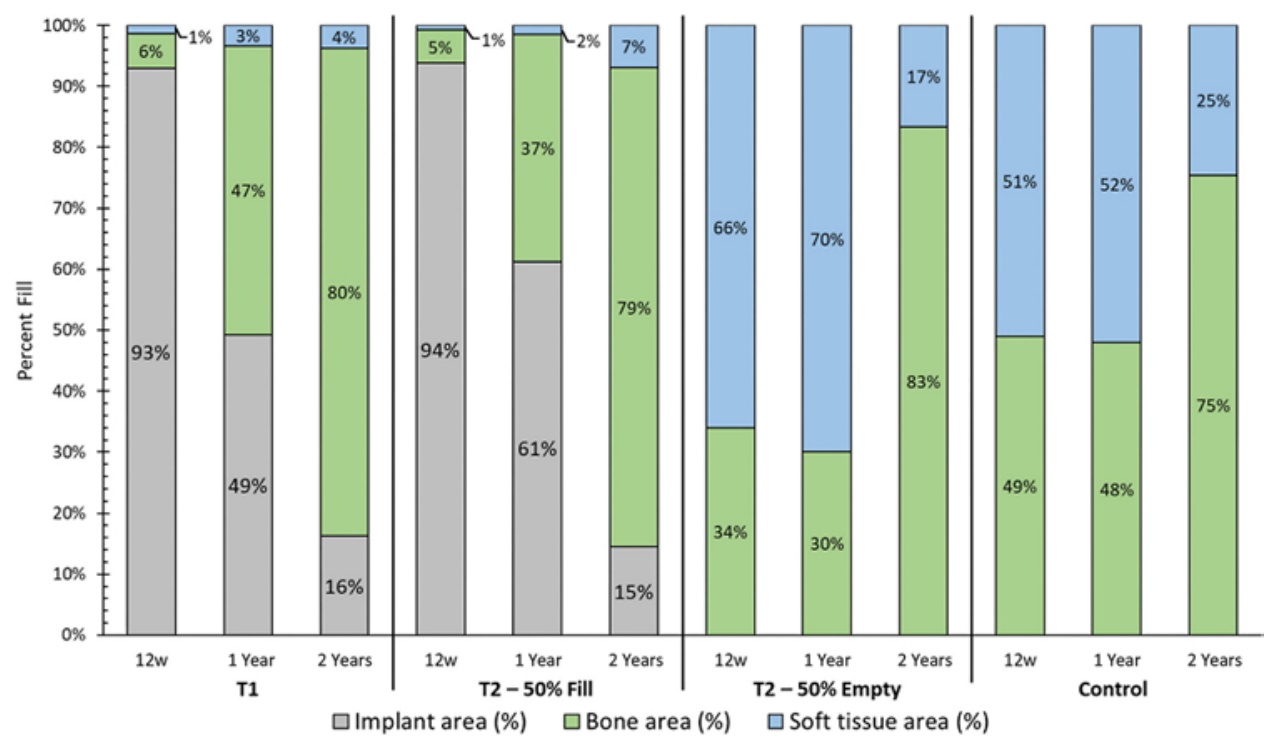

FIG. 5. Histomorphometric calculation of the relative amount of soft tissue (blue), bone (green), and implant (gray) in each kerf.

while bone and implant dominated over $95 \%$ of the kerf lines in test group 1 at all timepoints. Similar results were shown in the $50 \%$ filled kerf lines in test group 2. In contrast, the last 3 bars of data show that the control group kerfs were more than $50 \%$ filled with soft tissue until 2 years, at which point bone replaced approximately half of it. The 50\% empty kerf lines in test group 2 showed results similar to those of the control group.

\section{Discussion}

\section{Clinical Observations}

While impaired hind leg movement was observed in the initial sheep treated in this study, this finding was no longer observed after changing the surgical position of the sheep from flexed hind legs to extended hind legs. Thus, the impaired motion was most likely not induced by TTCP-PS or plate-screw fixation but was due to intraoperative sheep positioning, which likely caused peripheral neuromuscular compression.

Aside from the initial sheep positioned with flexed hind legs, only 1 sheep in the study displayed abnormal neurological signs. These signs resolved after postoperative day 1. Significantly, CSF leakage was noted during this sheep's surgery, which implies that the trephine lacerated the dura and subarachnoid membrane. Interestingly, although this sheep did not undergo dural repair, the bone flap was secured and sealed with TTCP-PS. No further evidence of CSF leak was observed. One sheep that was found dead after 50 weeks had a parapharyngeal abscess, which was determined as the cause of death and was considered unrelated to the surgery or treatment.

\section{Mechanical Strength}

In this ovine model, TTCP-PS yielded better bone flap fixation strength and better bone healing than plate-andscrew fixation. The force needed to fracture a native human skull varies between 2000 and 12,500 N, depending on location in the cranium. Thus, the pushout strength provided by TTCP-PS in this study $(>3000 \mathrm{~N})$ would provide strength closer to that of the native skull than would hardware fixation. ${ }^{11,12}$

In this study, animals were not euthanized early to compare differences in flap fixation strength between the test groups and control groups before 12 weeks. However, prior (unpublished) testing in sheep cadaver skulls demonstrated that the condition in test group 1 (100\% kerf fill) produced greater initial fixation strength than did plates and screws. Interestingly, this initial strength was lower than the strength found in the current study at 12 weeks, which was itself lower than the strength at 1 year. We posit that these increases in strength over time are due to bony ingrowth (osteoconduction) and provide a large enough effect to eliminate statistically significant differences between test groups 1 and 2 .

Hardware loosening, including instances of screw migration, was observed in a majority of the control group sites at both the 1- and 2-year timepoints. Bone flaps fixated with TTCP-PS, in contrast, showed no evidence of material migration or flap dislodgment. Based on these observations, fixation of human cranial flaps with TTCPPS might eliminate cosmetic disfiguration and pain that patients may experience if the metal plates and screws holding their bone flaps in place loosen over time.

\section{Histology and Histomorphometry}

There were no signs of infection or cytotoxicity at the surgical site in any group throughout the study. The control kerfs demonstrated greater fibrosis at all timepoints than kerfs filled with TTCP-PS. Sites were healed so well in the TTCP-PS group by 1 year that they were difficult to distinguish from the surrounding skull during gross examination. The lack of fibrosis and a high degree of osteointegration in the kerfs treated with TTCP-PS resulted in one contiguous skull and a framework for healing with new bone. Soft tissue remained in the control group kerfs and 
hindered complete bone healing. The low degree of fibrosis, high level of osteointegration, and new bone growth in the TTCP-PS groups all indicate better healing performance of the kerfs fixed with TTCP-PS.

The findings of this study indicate that TTCP-PS provided greater fixation strength than plate-and-screw fixation in this ovine cranial bone flap model and also resulted in better and faster bone healing of the flap kerfs.

\section{Limitations and Future Work}

This study lacked an intentional durotomy; in human clinical cases, a durotomy is typically performed after the craniotomy to access the brain. The safety of TTCP-PS should be further evaluated in the presence of a durotomy to evaluate its effects on the compromised dura, possible CSF leakage, and the exposed brain below the incision. A neurotoxicity study should also be done to ensure the safety of TTCP-PS before its introduction into possible human clinical trials. In addition to testing TTCP-PS's safety in a typical craniotomy and durotomy model, its effectiveness should be evaluated in other cranial approaches (e.g., posterior fossa, transsphenoidal, supraorbital, and various skull base approaches).

Finally, the unique, wet-field adhesive properties of TTCP-PS enable it to fill and possibly even seal the craniotomy kerf and burr holes to prevent CSF leaks, even in the presence of blood or other fluids. This capability should be tested, as it has the potential to significantly improve patient outcomes by mitigating both CSF leaks and the frequently resulting infections.

Although there are several potential improvements that TTCP-PS bone adhesive could provide for human cranial fixation, it is not yet known whether this technology will work in humans and what its economic value might be. Additional translational studies, including studies in humans, will be necessary to help determine this. While pricing has not been determined for this product, given the low cost of goods to produce TTCP-PS, it is expected that procedures using it will cost no more than those utilizing cranial plates and screws and may actually cost less.

\section{Conclusions}

TTCP-PS was demonstrated to be safe and effective for cranial flap fixation in an ovine model. In this study, the bioresorbable bone adhesive appeared to have multiple advantages over standard plate-and-screw bone flap fixation, including biomechanical superiority, more complete and faster bony healing across the flap kerfs without fibrosis, and the minimization of bone flap and/or hardware migration and loosening. These properties of TTCP-PS may improve human cranial bone flap fixation and cranioplasty.

\section{Acknowledgments}

We thank the staff of Biomatech for their work and support of this animal study. This study was conducted at Biomatech (Lyon, France), an independent contract research organization that followed good laboratory practice (GLP) conditions to ensure the study utilized sound scientific methods for impartial data collection and comparison, including blinded observations for the histological examination and results reported. The biomechanical test- ing was conducted at the Biomatech facility by Stryker personnel trained to the test method. The TTCP-PS bone adhesive technology and the results from this study are assigned to LaunchPad Medical through an exclusive licensing arrangement between Stryker and LaunchPad Medical.

\section{References}

1. Cochran D, Jones A, Sugita R, Brown M, Guda T, Prasad $\mathrm{H}$, et al: Immediate dental implant stabilization in a canine model using a novel mineral-organic adhesive: four month results. Int J Oral Maxillofac Implants [in press], 2019

2. Dashti SR, Baharvahdat H, Spetzler RF, Sauvageau E, Chang SW, Stiefel MF, et al: Operative intracranial infection following craniotomy. Neurosurg Focus 24(6):E10, 2008

3. Donath K, Breuner G: A method for the study of undecalcified bones and teeth with attached soft tissues. The SägeSchliff (sawing and grinding) technique. J Oral Pathol 11:318-326, 1982

4. Farrar DF: Bone adhesives for trauma surgery: a review of challenges and developments. Int J Adhes Adhes 33:89-97, 2012

5. Gupta R, Adeeb N, Griessenauer CJ, Moore JM, Patel AS, Thomas AJ, et al: Removal of symptomatic titanium fixation plates after craniotomy. Acta Neurochir (Wien) 158:18451848, 2016

6. Kumar A, Maartens NF, Kaye AH: Evaluation of the use of BioGlue in neurosurgical procedures. J Clin Neurosci 10:661-664, 2003

7. Kwinta BM, Krzyżewski RM, Kliś KM, Donicz P, Gackowska M, Polak J, et al: Emergency reoperations in cranial neurosurgery. World Neurosurg 105:749-754, 2017

8. Lindner D, Schlothofer-Schumann K, Kern BC, Marx O, Müns A, Meixensberger J: Cranioplasty using custom-made hydroxyapatite versus titanium: a randomized clinical trial. J Neurosurg 126:175-183, 2017

9. Moles A, Heudes PM, Amelot A, Cristini J, Salaud C, Roualdes V, et al: Long-term follow-up comparative study of hydroxyapatite and autologous cranioplasties: complications, cosmetic results, osseointegration. World Neurosurg 111:e395-e402, 2018

10. Mollman HD, Haines SJ: Risk factors for postoperative neurosurgical wound infection. A case-control study. J Neurosurg 64:902-906, 1986

11. Motherway JA, Verschueren P, Van der Perre G, Vander Sloten J, Gilchrist MD: The mechanical properties of cranial bone: the effect of loading rate and cranial sampling position. J Biomech 42:2129-2135, 2009

12. Siswanto A, Chong S: Strength analysis of human skull on high speed impact. Int Rev Mech Eng 6:1508-1514, 2012

13. Stoker MA, Forbes JA, Hanif R, Cooper C, Nian H, Konrad PE, et al: Decreased rate of CSF leakage associated with complete reconstruction of suboccipital cranial defects. J Neurol Surg B Skull Base 73:281-286, 2012

\section{Disclosures}

Funding for this study was provided by Stryker. Drs. Foley, Woodard, and Slotkin are consultants to LaunchPad Medical. Mr. Hess, Mr. Brown, and Ms. Baldwin are employees of LaunchPad Medical. Mrs. Mayotte is an employee of Stryker. Other associations include the following. Dr. Foley: consultant for Medtronic; direct stock ownership in Digital Surgery Systems, Discgenics, DuraStat, Medtronic, NuVasive, nView Medical, Practical Navigation/Fusion Robotics, SpineWave, TDi, and Triad Life Sciences; patent holder with Medtronic and NuVasive; royalties from Medtronic; board of directors of Digital Surgery Systems, Discgenics, DuraStat, LaunchPad Medical, nView Medical, Practical Navigation/Fusion Robotics, TDi, and Triad Life Sciences. Dr. 
Woodard: ownership in LaunchPad Medical. Dr. Slotkin: direct stock ownership in LaunchPad Medical. Ms. Baldwin: employee of LaunchPad Medical. Mr. Brown: employee and stockholder in LaunchPad Medical. Mr. Hess: ownership in LaunchPad Medical.

\section{Author Contributions}

Conception and design: Hess, Mayotte. Acquisition of data: Hess, Mayotte. Analysis and interpretation of data: all authors. Drafting the article: Hess, Foley, Woodard, Slotkin, Baldwin, Brown. Critically revising the article: all authors. Reviewed submitted version of manuscript: Hess, Foley, Woodard, Slotkin, Baldwin,
Brown. Approved the final version of the manuscript on behalf of all authors: Hess. Statistical analysis: Hess, Baldwin, Brown. Administrative/technical/material support: Hess. Study supervision: Hess.

\section{Correspondence}

Brian J. Hess: LaunchPad Medical, Lowell, MA. bhess@ launchpadmedical.com. 